Progress in Flight Physics 5 (2013) 425-440

DOI: $10.1051 /$ eucass/201305425

(C) Owned by the authors, published by EDP Sciences, 2013

\title{
EVALUATION OF THE AERODYNAMIC PROPERTIES OF THE INTERMEDIATE EXPERIMENTAL VEHICLE IN THE RAREFIED AND TRANSITIONAL REGIME
}

\author{
T. Bányai ${ }^{1}$, E. Torres $^{1}$, T. E. Magin ${ }^{1}$, A. V. Kashkovsky ${ }^{2}$, \\ P. V. Vashchenkov ${ }^{2}$, M. S. Ivanov ${ }^{2}$, and P. Rambaud ${ }^{1}$ \\ ${ }^{1}$ von Karman Institute for Fluid Dynamics \\ Rhode-St-Genese, Belgium \\ ${ }^{2}$ Khristianovich Institute of Theoretical and Applied Mechanics \\ Siberian Branch of the Russian Academy of Sciences \\ Novosibirsk, Russia
}

\begin{abstract}
In order to evaluate the behavior of the intermediate experimental vehicle (IXV) in the upper layer of the atmosphere, series of computations were carried out by means of the Direct Simulation Monte Carlo (DSMC) method, which are reported hereby. First an introduction is given about the IXV mission followed by a short explanation on DSMC and the computational methodology. A first validation case is demonstrated for computations based on the geometry of the Apollo capsule, showing good agreement with a reference in literature. Then, simulations of the IXV are presented, including flow-thruster interaction. Finally, the result matrix of aerodynamic properties is shown.
\end{abstract}

\section{INTRODUCTION}

The objective of the ESA FLPP program is to position Europe as a key player in the strategic area of atmospheric reentry which is of outmost importance for future international transportation, exploration and scientific development. Europe needs to reach a level of technological maturity in order to engage in development of an efficient Reusable Space Transportation System. This need served as the driving force in the establishing of the IXV mission. The draft of the mission is envisioned as launching from Kourou (French Guiana) on a VEGA rocket to reach an orbit of about $180 \times 300 \mathrm{~km}$. VEGA's upper stage will trigger the reentry which formally begins around $120 \mathrm{~km}$ with $7700 \mathrm{~m} / \mathrm{s}$ and

This is an Open Access article distributed under the terms of the Creative Commons Attribution License 2.0, which permits unrestricted use, distribution, and reproduction in any medium, provided the original work is properly cited. 
-1.19 degree path angle. Landing is foreseen to be a splash down in the Pacific Ocean. A detailed explanation and overview can be found in [1] and [2].

At high altitudes, where the scales of the molecular level are comparable with the scales of the macroscopic properties, fluid models based on continuum representation (i. e., Navier-Stokes equations) fail to describe the flow accurately. At such conditions, the DSMC method is used for solving the Boltzmann equation. At von Karman Institute for Fluid Dynamics (VKI), the software employed for DSMC-type simulations is the so-called Rarefied Gas Dynamics Analysis System (RGDAS), which is developed at the Russian Academy of Sciences, Siberian Branch, Khristianovich Institute of Theoretical and Applied Mechanics [3] and has been applied in numerous investigations (see, for example, [4-8]).

The main focus of this work is to evaluate the aerodynamic properties of IXV in the rarefied and transitional region by means of DSMC simulations. First, a validation is presented against Moss et al. [9] for the Apollo capsule. It is carried out by comparing aerodynamic coefficients on a series of test cases at different points of trajectory and angles of attack. Second and most importantly, the simulations of IXV are presented for the aerodynamic coefficients along the descent as low as the VKI computational facility allows. To assess the stability of IXV similar to the study of Apollo, a test case matrix will be computed at different altitudes and angles of attack. Finally, a brief study is shown for the joint simulation of flow around IXV while stabilizing thrusters are on.

\section{DIRECT SIMULATION MONTE CARLO METHOD}

Direct simulation Monte Carlo method [10] is a particle simulation method based on the kinetic theory of gases and is typically employed to study rarefied flows. The main principle of DSMC is the splitting of continuous process of molecular motions and collisions into two successive stages at the time step $\Delta t$. The computational domain is divided into cells of size $\Delta x$ such that the variation of the flow parameters in every cell is small. The time step $\Delta t$ should be small comparing to the mean collision time $\tau_{\lambda}$. Free motion of molecules and their collisions are considered successively at each time step. Collisions of particles are carried out independently within each cell in the physical space, i. e., the collisions of particles in the neighboring cells are not considered. Since the distribution function variation is supposedly small in the cell, when a pair of particles is chosen for the collision the relative distance between them is not taken into account. The postcollision velocities are calculated in accordance with the conservation laws of linear momentum and energy. All molecules located in the computational domain are displaced by a distance determined according to their instantaneous velocities and the time step $\Delta t$. At the same time, new particles are introduced at inlets, the gas-surface interaction is taken into account at the walls, and parti- 
cles are eliminated at the exits of the computational domain. Thus, the following principal stages are specified in the DSMC calculation procedure:

- molecular motion;

- indexing of molecules over collision cells;

- collisions;

- entering new molecules;

- gas/surface collisions; and

- sampling of macroparameters.

As the simulation evolves by integrating over a sufficient period of time, distribution of macroscopic parameters can be evolved in the flow domain and on surfaces immersed into the flow. From the variety of the available codes (see RGDAS [11], DS2V/3V [12], MONACO [13], DAC [14], and DSMCFoam [15]), the software employed for DSMC at VKI is the Rarefied Gas Dynamics Analysis System code (RGDAS) in collaboration with its developers at the Russian Academy of Sciences, Siberian Branch, Khristianovich Institute of Theoretical and Applied Mechanics.

\section{METHODOLOGY OF THE COMPUTATIONS}

The user interface of RGDAS offers a straightforward step-by-step procedure for setting up the test cases. The computations presented in this article are carried out by following the guidelines explained in this section. Details and numerical parameters are given separately for Apollo and IXV in the following sections.

Collision and chemistry: At first, the "flow type" needs to be specified in terms of collisional model and chemical interactions. The Variable Hard Sphere (VHS) model has been chosen with continuous internal energy for collisions and five species $\left(\mathrm{N}, \mathrm{O}, \mathrm{N}_{2}, \mathrm{NO}\right.$, and $\left.\mathrm{O}_{2}\right)$ reaction set (labeled as Air) for chemistry. The required parameters related to the collision and chemistry (for example, molecular mass, VHS diameter, etc.) have been kept at preset values corresponding to Air.

Surface definition: Next, the surface approximation of the subject vehicle have to be imported into the RGDAS environment. For IXV, a CAD model was provided by Dassault Aviation which then was cleaned, simplified and meshed by means of Gambit software. The geometry of Apollo has also generated and meshed in Gambit, according to the specifications in [9]. Finally, both meshes 
were checked for integrity (closedness, facing-out normal vectors, etc.) and converted into the format accepted by RGDAS. Constant temperature noncatalytic wall boundary conditions were applied both for Apollo and IXV.

Computational grid: The choice of the computational domain greatly affects the quality of the solution. Since RGDAS is based on adaptive Cartesian mesh (automatic) refinement, the right choices are needed to supply for the $X$, $Y, Z$ dimensions of the domain and the unrefined grid size (called background grid size). On the upstream side, a clearance of at least $0.5 \mathrm{~m}$ was kept in front of the shock. The driving requirement for the downstream side of the domain was to ensure a supersonic outlet, avoiding any information to travel backwards. The target mesh resolution was to set the background grid size equal to the free stream mean free path. However, from $100 \mathrm{~km}$ and above, the background grid size was kept at the value corresponding to $100 \mathrm{~km}$, in order to allow the macroscopic parameter sampling to capture the flow structure. A proper choice of the grid size was confirmed by comparing the adapted grid size and the resulting mean free path.

Flow parameters: Specification of the flow parameters involved providing the free stream conditions, namely: angle of attack (AoA), translational temperature $\left(T_{\infty}\right)$, velocity $\left(V_{\infty}\right)$, number density $\left(n_{\infty}\right)$, molar composition of the species $i\left(X_{\infty i}\right)$, and, finally, the rotational and vibrational temperature for the diatomic molecules (set to the translational temperature).

Numerical parameters: DSMC is a particle method and, contrary, to solvers based on Eulerian methods, it does not diverge by nature, leading to the fact that results can be rendered unusable if improper settings were used. Therefore, appropriate values of the numerical parameters are crucial, and their estimation can be summarized as follows:

- the time step size $\Delta T$ was chosen such that the particles do not cross more than one collisional cell on the background grid size based on the free stream velocity. For reentry calculations, this has proven to be a satisfactory choice, since the automatic grid refinement mostly occurs at high density regions, where the flow is substantially decelerated. After the computation was completed, the choice of the $\Delta T$ was verified through the results;

- the ratio between real molecules and model particles $\left(F_{\text {num }}\right)$ was chosen such that the number of particles per cell is about 8 in the free stream [16];

- the number of time steps before sampling $\left(N_{\text {start }}\right)$ is equal to $5-10$ flowthrough (the number of time steps required for a particle to pass through the domain with an average speed), estimated by preliminary/previous computations started with sampling and verified by monitoring the evolution of intermediate parameters such as the number of cells and particles; and 


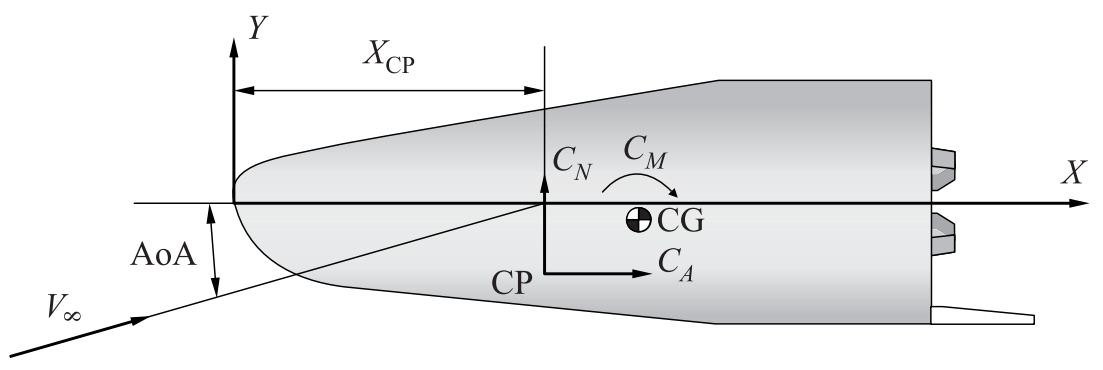

Figure 1 Definition of aerodynamic properties

- the number of sampling time steps $\left(N_{\text {sampling }}\right)$ : 10-15 flowthrough, the period extended on an on-demand basis if convergence of the results is unsatisfactory.

Interpretation of the results: the present work is focused on the estimation of the aerodynamic coefficients in the body-fitted coordinate system and followed the nomenclature and sign convention shown in Fig. 1, where quantities $C_{A}$ and $C_{N}$ are the axial and normal force coefficients, respectively; $C_{M}$ is the pitching moment coefficient; $X_{\mathrm{CP}}$ is the $X$ coordinate of the center of pressure (CP); and CG is the center of gravity. Free stream parameters are distinguished by subscript $\infty$.

\section{APOLLO}

The extensive and well-documented work carried out by Moss et al. [9, 17] on the Apollo capsule by means of DSMC (DS3V [18]) and Navier-Stokes (LAURA [19]) codes proved to be an ideal reference test case for validating the methodology of the computations employed at the VKI. In the present study, the behavior at several points of the reentry trajectory have been recomputed and investigated. Two main comparisons have been performed, one at constant angle of attack with a varying altitude between 200 and $85 \mathrm{~km}$ and one more comparison at constant altitude with a varying AoA between $0^{\circ}$ to $\pm 180^{\circ}$. Axial and normal force coefficients, the pitching moment coefficient and $X$ coordinate of the center of pressure have been compared against reference quantities.

\subsection{Conditions}

Fortunately, all the data required for the DSMC computations were directly available from [9]. Table 1 shows the atmospheric conditions and the capsule's 
Table 1 Free stream conditions and wall temperature in function of altitude for the Apollo capsule

\begin{tabular}{cccrrrrc}
\hline$H, \mathrm{~km}$ & $\mathrm{Kn}$ & $n_{\infty}, 1 / \mathrm{m}^{3}$ & $T_{\infty}, \mathrm{K}$ & $T_{w}, \mathrm{~K}$ & $X_{\mathrm{O}_{2} \infty}$ & $X_{\mathrm{N}_{2} \infty}$ & $X_{\mathrm{O} \infty}$ \\
\hline 200 & 44.74 & $8.999 \cdot 10^{15}$ & 1026 & 234 & 0.0314 & 0.4547 & 0.5137 \\
150 & 7.59 & $5.305 \cdot 10^{16}$ & 733 & 373 & 0.0546 & 0.6155 & 0.3298 \\
130 & 2.07 & $1.942 \cdot 10^{17}$ & 500 & 524 & 0.0708 & 0.6911 & 0.2379 \\
115 & 0.408 & $9.856 \cdot 10^{17}$ & 304 & 795 & 0.0977 & 0.7538 & 0.1483 \\
110 & 0.190 & $2.124 \cdot 10^{18}$ & 247 & 920 & 0.1232 & 0.7704 & 0.1063 \\
105 & 0.081 & $5.094 \cdot 10^{18}$ & 208 & 1029 & 0.1580 & 0.7831 & 0.0587 \\
95 & 0.0139 & $3.116 \cdot 10^{19}$ & 189 & 1295 & 0.2004 & 0.7868 & 0.0127 \\
85 & 0.0024 & $1.654 \cdot 10^{20}$ & 181 & 1598 & 0.2372 & 0.7628 & 0.0000 \\
\hline
\end{tabular}

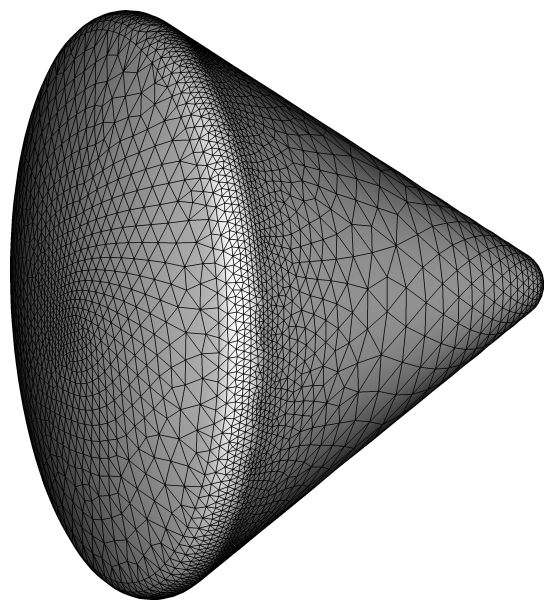

Figure 2 Surface mesh of the Apollo capsule wall temperature in function of altitude. In all computations, the free stream velocity was set to $9.6 \mathrm{~km} / \mathrm{s}$. For the descent study, the altitudes considered were 200, 150, 130, 115, 110, 100, 95, and $85 \mathrm{~km}$ with AoA $=-25^{\circ}$. The rotation study was performed with 15 degree increments at the altitude of $105 \mathrm{~km}$. The surface approximation is composed of 8217 triangular elements, shown in Fig. 2.

The computations were carried out on VKI SGI Altix ICE 8200 dual plane cluster (64 blades equipped with 128 quad cores Xeon processors at $2.5 \mathrm{GHz} / 12 \mathrm{M} / 1333 \mathrm{MHz}$, with 256 set of $2 \times 2$ GB DIMM $667 \mathrm{MHz}$ and Infiniband connection).

\subsection{Results}

Regarding the constant altitude computations (depicted in Fig. 3), very close agreement can be observed, even in spite of the fact that different solvers were employed.

In the case of study along the trajectory, at low altitudes, there are significant deviations between the reference and VKI computations. The reason is 


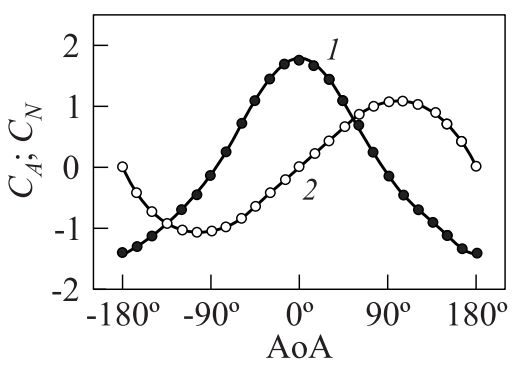

(a)

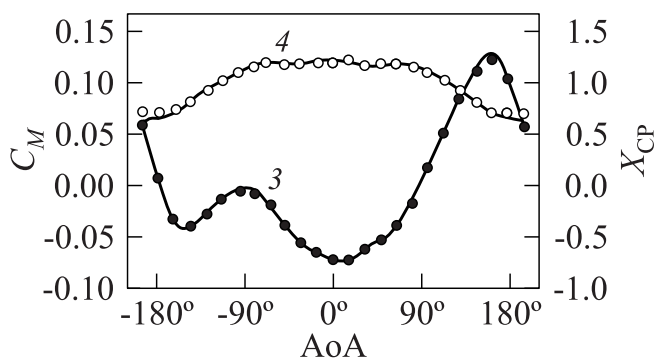

(b)

Figure 3 Evolution of axial (1) and normal (2) force coefficients (a) and moment coefficient (3) and axial position of $\mathrm{CP}(4)(b)$ as a function of AoA at constant altitude of $H=105 \mathrm{~km}$. Curves — results of [9], signs — VKI computations

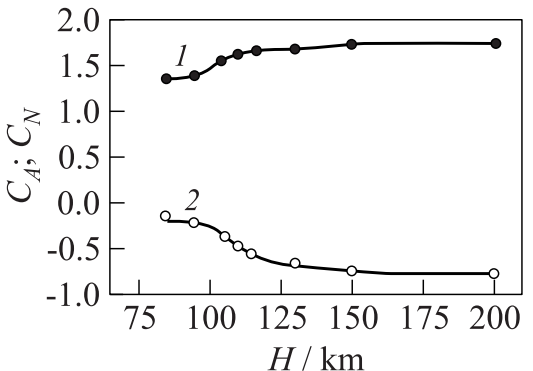

(a)

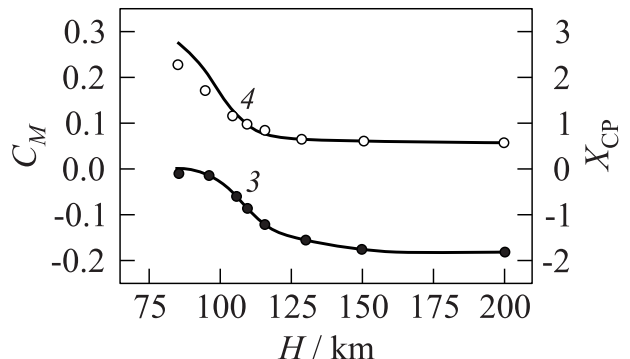

(b)

Figure 4 Evolution of axial (1) and normal (2) force coefficients (a) and moment coefficient (3) and axial position of CP $(4)(b)$ as a function of altitude at constant angle of attack AoA $=-25^{\circ}$. Curves - results of [9], signs - VKI computations

attributed to a higher resolution in the case of the VKI calculation. For example, in the 85-kilometer case, the reference calculation contains $\sim 11$ million particles, while for the VKI counterpart it was about 600 million. However, still with this large amount of particles - due to the limitation in computational resources - the solution is slightly unresolved (background grid size is about 1.6 times the free stream mean free path).

An additional study has been performed to assess the sensitivity against the most important numerical parameters (number of particles per cell and background grid size). Three different altitudes were considered, each inheriting remarkably different flow structure. At $200 \mathrm{~km}$, the flow is rarefied. At $95 \mathrm{~km}$, the flow is just at the edge between the transitional and continuum regimes. At $110 \mathrm{~km}$, an intermediate altitude is selected. For each altitude, a matrix of cases with 4-5 different mesh resolutions and 3 sets of particles $(5,10$, and 15$)$ were 


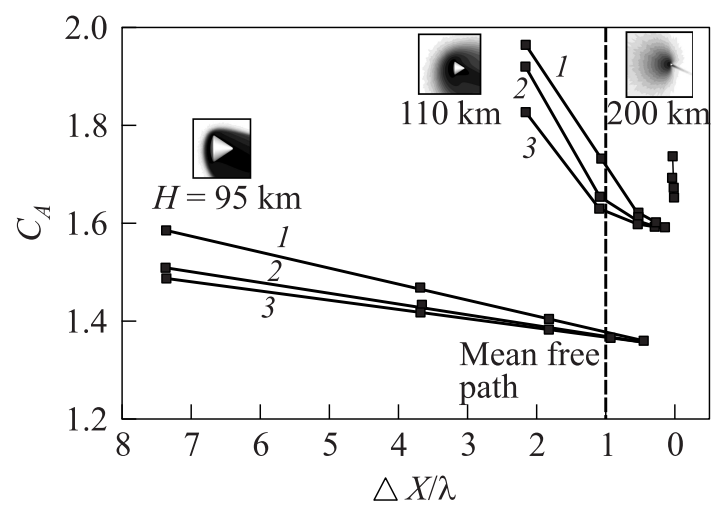

Figure 5 Sensitivity study of axial force coefficient vs. background grid size normalized by the free stream mean free path in function of number of particles per cell $(1-$ $5 ; 2-10$; and $3-15$ ) at altitudes $H=95,110$, and $200 \mathrm{~km}$. Dashed vertical line shows the location where the background grid size equals to the mean free path

computed. Figure 5 summarizes the results. For the 200- and 110-kilometer case, convergence was found, although for the 200-kilometer case, the resolution had to be kept high (but the actual $\Delta X$ was still larger or comparable to the 110-kilometer case) in order to resolve the flow close to the body. In case of $95 \mathrm{~km}$, no conventional convergence is observed, however, the difference between $\Delta X=\lambda$ and extrapolation to infinitely fine mesh reveals a difference of about $1 \%-1.7 \%$. The following behavior is expected:

- on finer grids the slope may flatten out; and

- at lower altitudes, the slope of the convergence decreases, further reducing the error.

To verify this behavior, ongoing simulations are in progress. These computations are extremely challenging in terms of computational resources.

\section{INTERMEDIATE EXPERIMENTAL VEHICLE}

The aim of the IXV computations was to establish a matrix of aerodynamic coefficients by means of the DSMC method from the early part of the reentry trajectory (which is at $120 \mathrm{~km}$ ) till as low as the computational facility allows (85 km currently). Intermediate experimental vehicle is a lifting body vehicle, and, therefore, target angles of attack between $0^{\circ}$ and $90^{\circ}$ were considered. Besides, the effect on the flow of the reaction control system (RCS) was investigated in the case of controlling pitching motion by activating the two bottom thrusters. 
Table 2 Free stream conditions in function of altitude for the IXV

\begin{tabular}{ccccccc}
\hline$H, \mathrm{~km}$ & $\mathrm{Kn}$ & $n_{\infty}, 1 / \mathrm{m}^{3}$ & $T_{\infty}, \mathrm{K}$ & $X_{\mathrm{O}_{2} \infty}$ & $X_{\mathrm{N}_{2} \infty}$ & $X_{\mathrm{O} \infty}$ \\
\hline 120 & 0.743 & $5.154 \cdot 10^{17}$ & 359 & 0.086 & 0.729 & 0.180 \\
115 & 0.390 & $9.811 \cdot 10^{17}$ & 299 & 0.100 & 0.749 & 0.146 \\
110 & 0.179 & $2.136 \cdot 10^{18}$ & 243 & 0.122 & 0.764 & 0.106 \\
105 & 0.0763 & $5.029 \cdot 10^{18}$ & 209 & 0.151 & 0.773 & 0.066 \\
100 & 0.0321 & $1.194 \cdot 10^{19}$ & 195 & 0.179 & 0.775 & 0.035 \\
95 & 0.0131 & $2.917 \cdot 10^{19}$ & 188 & 0.198 & 0.776 & 0.014 \\
90 & 0.00539 & $7.118 \cdot 10^{19}$ & 186 & 0.207 & 0.779 & 0.003 \\
85 & 0.00224 & $1.709 \cdot 10^{20}$ & 188 & 0.209 & 0.780 & 0.000 \\
\hline
\end{tabular}

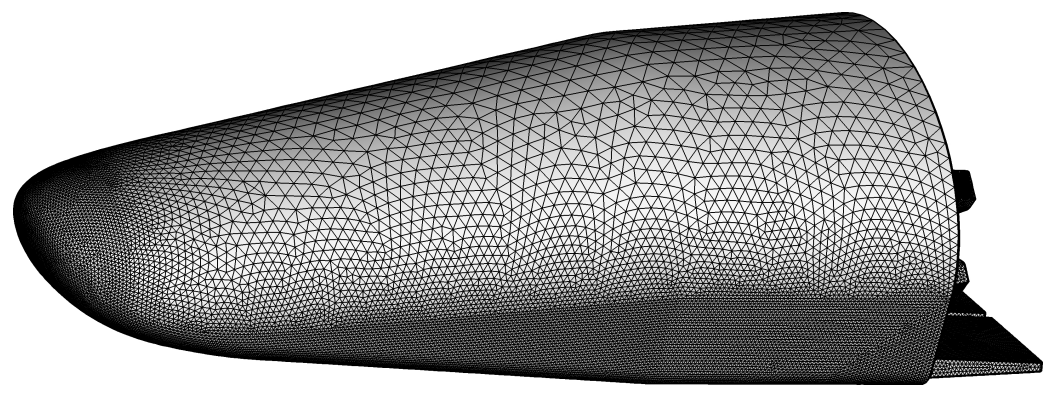

Figure 6 Surface mesh of the IXV

\subsection{Conditions}

All data required for the DSMC computations were provided by Dassault Aviation except the atmospheric conditions, which then were taken from [20] (Table 2). In all computations, the free stream velocity and the wall temperature was set to $7.45 \mathrm{~km} / \mathrm{s}$ and $900 \mathrm{~K}$, respectively. The reference length was $4.4 \mathrm{~m}$, and the moment reference point was set to $(2.552,-0.11,0) \mathrm{m}$ as shown in Fig. 1. The surface approximation was composed of 122,510 triangular elements shown in Fig. 6. The matrix was built up by 5-kilometer decrements in altitude and 15 degree increments in AoA. For all computations, the flap deflection angle was set to $0^{\circ}$.

\subsection{Results}

Fortunately, very little technical difficulties remain unclarified (compared to the Apollo simulation campaign) on how to carry out the IXV computations. Sim- 


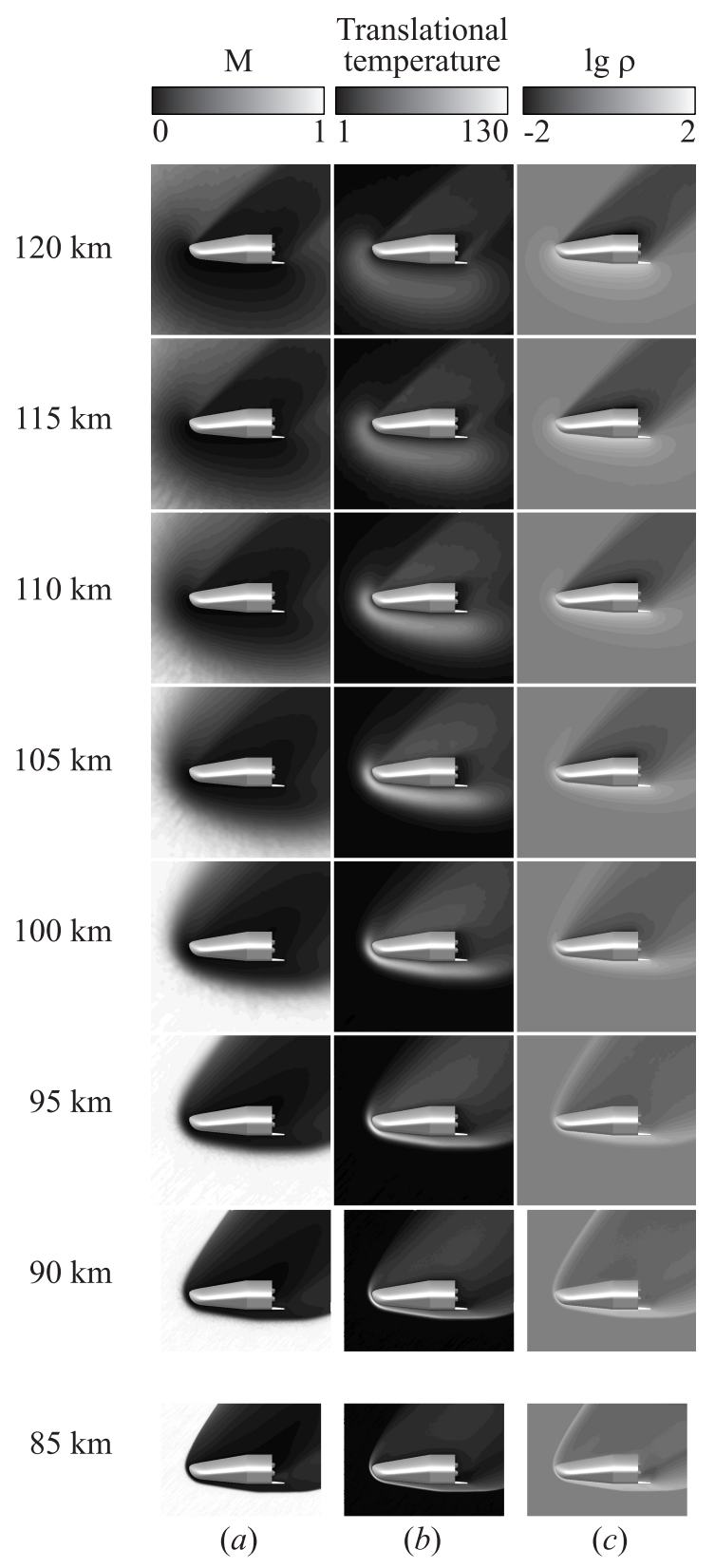

Figure 7 Contour plots of the flow properties in the midplane for the $\mathrm{AoA}=45^{\circ}$ cases along the trajectory: $(a)$ free stream normalized Mach number; $(b)$ translational temperature; and $(c)$ density 

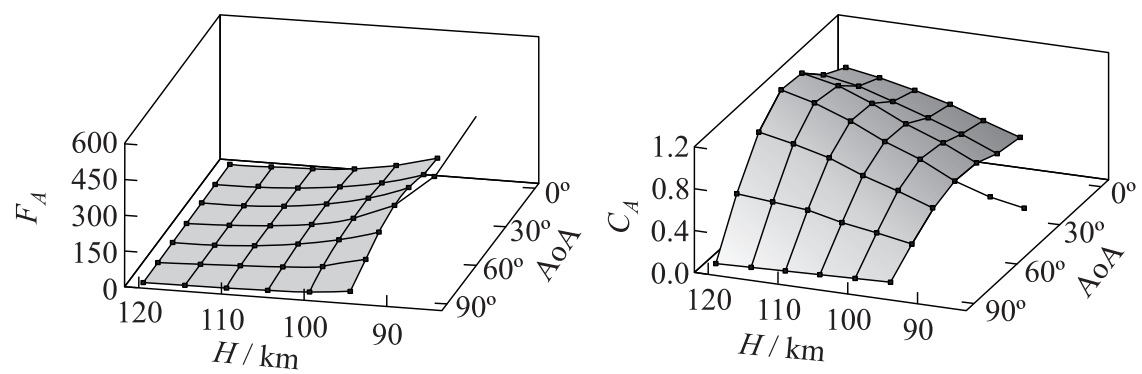

(a)
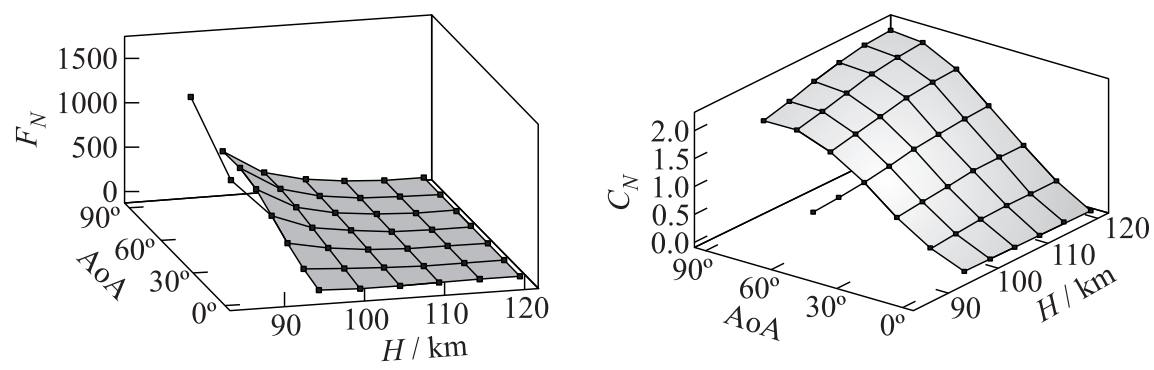

(b)

Figure 8 Axial force and its coefficient $(a)$ and normal force and its coefficient $(b)$ vs. altitude and AoA. Result matrix for the IXV

ilarly to the corresponding Apollo calculations, the 85-kilometer case proved to be too large for the computational resources available at VKI; therefore, the background grid size had to be increased to 1.6 times the size of the free stream mean free path (resulting in 782 million particles). Due to this demand on hardware and available CPU time, only the 45 degree AoA case has been computed for altitudes of 85 and $90 \mathrm{~km}$. Figure 7 shows the evolution of the free stream normalized Mach, temperature, and density fields in function of altitude:

- at $120 \mathrm{~km}$, a very weak shock can be observed. Following the path down on the trajectory, the shock becomes stronger and thinner, while at the same time, the density and temperature increase on it; and

- the wake also becomes increasingly encapsulated by the shock.

Figures 8 and 9 summarize the aerodynamic quantities. Left column shows the actual values of the axial and normal forces and the pitching moment, while the right column shows the corresponding coefficients. Regarding the behavior of the vehicle, the following observations can be stated: 


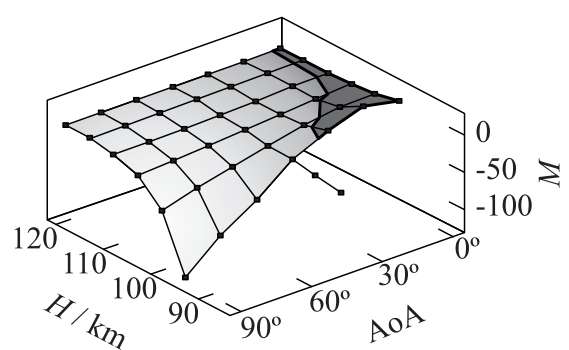

(a)

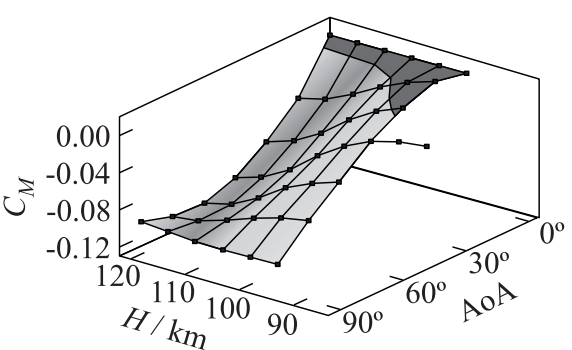

(b)

Figure 9 Pitching moment $(a)$ and its coefficient $(b)$ vs. altitude and AoA. Result matrix for the IXV. Darkened region indicates positive moment

- the results indicate that the vehicle is stable in the examined region;

- at higher altitudes, the neutral angle of attack is close to zero and converges to the nominal 45 degree AoA while advancing down on the trajectory;

- due to the density increase, the axial and normal forces build up dramatically but the moment remains close to zero (slightly increases), ensuring a stable transition (throughout the examined altitude range) into the more dense atmosphere;

- around $100 \mathrm{~km}$ or higher, the moment arising from the aerodynamic forces may not be enough to successfully counteract the vehicle's inertia gained by a disturbance; and

- the nominal AoA is close to the axial force maximum at all examined altitudes.

\subsection{Activation of the Reaction Control System}

In the rarefied and uppertransitional regime, the aerodynamic forces and moments are still relatively low for exploiting maneuvering by means of control surfaces. Therefore, maneuvering needs to be carried out via thrusters belonging to RCS. The gas exiting from such a thruster is well in the continuum regime, rendering the problem impossible to solve purely by DSMC method at currently available computational resources. A coupled Navier-Stokes-DSMC solution strategy is required $[7,21,22]$ where the core of the jet is computed by a continuum solver and serves as boundary condition for DSMC at the border 
Table 3 Properties of the thruster jet in the nozzle exit cross section

\begin{tabular}{cccccc}
\hline$F, \mathrm{~N}$ & $A, \mathrm{~m}^{2}$ & $m, \mathrm{~kg} / \mathrm{s}$ & $\rho, \mathrm{kg} / \mathrm{m}^{3}$ & $V, \mathrm{~m} / \mathrm{s}$ & $T, \mathrm{~K}$ \\
\hline 380 & 0.006104 & 0.153 & 0.0097 & 2540 & 371 \\
\hline
\end{tabular}

between the continuous and rarefied regions. Unfortunately, the jet is embedded in a perpendicular flow, forcing this interface to be kept submerged in the boundary layer, which includes computational requirements beyond our current computational resources. Therefore, core parts of the jet had to be simulated in an unresolved fashion in terms of collisional grid. In the current case, the thruster was modeled as a pure nitrogen jet with the parameters listed in Table 3 at the nozzle exit. The effect at altitude $94.43 \mathrm{~km}$ was investigated where the two lower thrusters were fired, each providing a thrust of around $380 \mathrm{~N}$. At this altitude the density of the atmosphere is so low that the plume penetrates the free stream like a solid spear and starts to bend slowly, which is illustrated in Fig. 10. Changes in coefficients are reviewed in Table 4. The forces do not

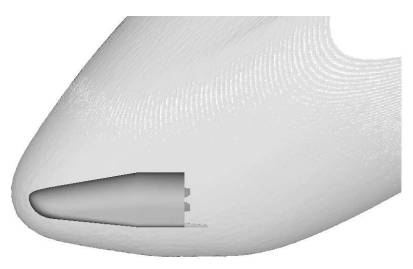

(a)
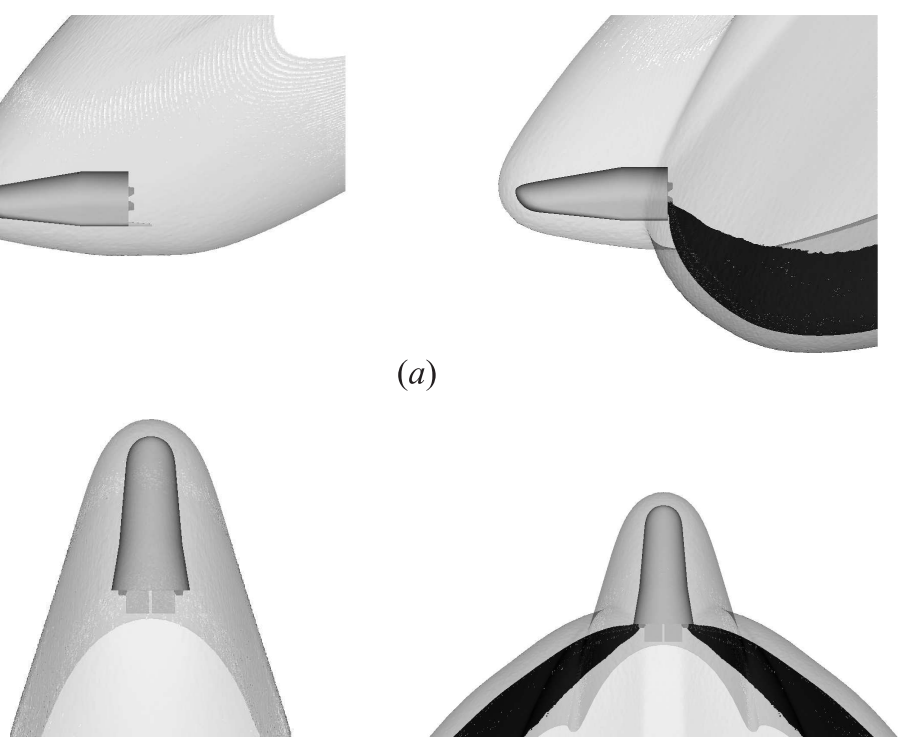

(b)

Figure 10 Side $(a)$ and top (b) views of flow around IXV at $94.43 \mathrm{~km}$ : without (left column) and with (right column) two downward thrusters activated; ISO-surface plots of $\mathrm{M}=10$ and $X_{\mathrm{N}_{2}}=0.92$ 
Table 4 Aerodynamic quantities (forces, moments, and their coefficients) of the IXV at altitude of $94.43 \mathrm{~km}$, with and without activation of the two lower boosters of the RCS

\begin{tabular}{ccccccc}
\hline Regime & $F_{A}, \mathrm{~N}$ & $F_{N}, \mathrm{~N}$ & $M, \mathrm{~N} \cdot \mathrm{m}$ & $C_{A}$ & $C_{N}$ & $C_{M}$ \\
\hline RCS on & 153.4906 & 356.3175 & -25.5388 & 0.4760 & 1.1050 & -0.0180 \\
RCS off & 156.7152 & 351.8030 & -17.5934 & 0.4860 & 1.0910 & -0.0124 \\
\hline
\end{tabular}

change noticeably, but the increase in moment is significant. However, this increase is about $1 \%-2 \%$ of the moment generated by the thrusters at this altitude. Further study for addressing this particular setup was carried out in [8].

\section{SUMMARY}

The present paper summarized the ongoing DSMC activities of VKI in relation to hypersonic reentry of the IXV. An overview was given on the mission of IXV and the DSMC technique followed by the methodology employed at VKI for rarefied flow computations. A short overview was given about RGDAS, the software used for this task. Validation of the VKI methodology for the computation was carried out for the Apollo capsule against reference data available in the literature. Results show good agreement with a slight deviation for the lower altitude computations. Based on the knowledge and experiences of the Apollo computations, a similar study was carried out for the IXV in order to support the aerothermodynamic database. The lower limit was reached at the altitude where it was still possible to perform near to resolved simulations. This limit turned out to be around $85 \mathrm{~km}$ at the time of the writing of this paper. Currently, the main focus regarding calculations by means of the DSMC method is to push the limit of RGDAS, allowing proper computations as low as possible in altitude. Finally, a calculation with activated maneuvering thrusters was presented to assess the feasibility of a coupled Navier-Stokes-DSMC computation. Experiences on simulation with RCS led to the same milestone as the computations in the transitional (near continuum) regime: main textitasis needs to be put on the availability of the low Knudsen number computations.

\section{ACKNOWLEDGMENTS}

The authors acknowledge the following persons and organizations for supporting this work: 
- Dassault Aviation: Jean-Pierre Tribot and Sylvain Dutheil;

- ThalesAlenia Space: Vincenzo Mareschi;

- Siberian Branch of Russian Academy of Sciences (Lavrentyev Youth Grant "High-altitude aerothermodynamics of advanced spacecraft taking into account nonequilibrium chemical reactions"); and

- Russian Foundation for Basic Research (Grants No.10-08-01203 and No. 11-01-91162)

We gratefully acknowledge Tiago Quintino and Francesco Panerai for useful discussions.

\section{REFERENCES}

1. Tumino, G., and Y. Gerard. 2006. IXV: The Intermediate eXperimental vehicle â Europe among the world players in atmospheric reentry. In: ESA bulletin 128. 62-67.

2. Baiocco, P., S. Guedron, P. Plotard, and J. Moulin . 2006. The pre-X atmospheric re-entry experimental lifting body: Program status and system synthesis. 57th IAC Congress. Spain.

3. Ivanov, M. S. 2010. User Manual for Rarefied Gas Dynamics Analysis System (RGDAS).

4. Antonov, S. G., M. S. Ivanov, A. V. Kashkovsky, and V. G. Chistolinov. 1991. Influence of atmospheric rarefaction on aerodynamic characteristics of flying vehicles. 17th Symposium (International) on Rarefied Gas Dynamics Proceedings. 522-30.

5. Markelov, G. N., A. V. Kashkovsky, and M. S. Ivanov. 2001. Aerodynamics of space station "Mir" during aeroassisted controlled descent. AIP Conference Proceedings 585(1):745-52.

6. Vashchenkov, P.V., A.V. Kashkovsky, G. N. Markelov, A.N. Krylov, and M. S. Ivanov. 2004. Influence of atmospheric rarefaction on aerodynamic characteristics of flying vehicles. European Congress on Computational Methods in Applied Sciences and Engineering.

7. Vashchenkov, P. V., A.N. Kudryavtsev, D. V. Khotyanovsky, and M.S. Ivanov. 2005. DSMC and Navier-Stokes study of backflow for nozzle plumes expanding into vacuum. Rarefied gas dynamics: 24th International Symposium on Rarefied Gas Dynamics. Ed. M. Capitelli. 762:355-60.

8. Kashkovsky, A. V., P. V. Vashchenkov, T. Bányai, and M. S. Ivanov. 2011. Modelling IXV control thruster plume-surface interaction. 4th European Conference for Aerospace Sciences. St. Petersburg, Russia.

9. Moss, J. N., C. E. Glass, and F. A. Greene. 2006. DSMC simulations of Apollo capsule aerodynamics for hypersonic rarefied conditions. AIAA Paper No. 20063577 . 
10. Bird, G. A. 1994. Molecular gas dynamics and the direct simulation of gas flows. Oxford engineering science ser. Clarendon Press.

11. Ivanov, M.S., A. V. Kashkovsky, S. F. Gimelshein, G. N. Markelov, A. A. Alexeenko, Ye. A. Bondar, G. A. Zhukova, S. B. Nikiforov, and P. V. Vaschenkov. 2006. SMILE System for 2D/3D DSMC computations. 25th Symposium (International) on Rarefied Gas Dynamics Proceedings. Russia.

12. Bird, G. A. 2005. The DS2V/3V Program Suite for DSMC calculations. Rarefied gas dynamics: 24th International Symposium on Rarefied Gas Dynamics. Ed. M. Capitelli. American Institute of Physics Conference ser. 762:541-46.

13. Lofthouse, A. J., I. D. Boyd, M. J. Wright. 2007. Effects of continuum breakdown on hypersonic aerothermodynamics. Phys. Fluids 19(2).

14. LeBeau, G. J. 2002. A user guide for the DSMC analysis code (DAC) software for simulating rarefied gas dynamic environments. Revision DAC97-G 4674.

15. Hauser, T., and J. Allen. 2007. FoamDSMC - a DSMC solver for rarefied flow applications based on OpenFOAM.

16. Bird, G.A. 2007. Sophisticated DSMC. Notes prepared for a short course at the DSMC07 meeting. New Mexico. http://www.gab.com.au/Resources/ DSMC07notes.pdf.

17. Moss, J. N., C. E. Glass, and F. A. Greene. 2006. Blunt body aerodynamics for hypersonic low density flows. 25th Symposium (International) on Rarefied Gas Dynamics Proceedings. Russia.

18. Bird, G. A. 2006. Visual DSMC program for three-dimensional flows. The DS3V Program User's Guide.

19. Cheatwood, F. M., and P. A. Gnoffo. 1996. User's Manual for the Langley aerothermodynamic upwind relaxation algorithm (LAURA). NASA TM 4674.

20. U.S. Standard Atmosphere. 1976. NASA TM X 74335.

21. Wu, J. S., Y.-Y. Lian, G. Cheng, R.P. Koomullil, and K.-C. Tseng. 2006. Development and verification of a coupled DSMC-NS scheme using unstructured mesh. J. Comput. Phys. 219(2):579-607.

22. La Torre, F., S. Kenjeres, C. R. Kleijn, and J.-L. P. A. Moerel. 2009. Evaluation of micronozzle performance through DSMC, Navier-Stokes and coupled DSMC/Navier-Stokes approaches. Computational Science - ICCS 2009. Lecture notes in computer science. 5544:675-84. 